International Mathematical Forum, Vol. 8, 2013, no. 28, 1369 - 1374

HIKARI Ltd, www.m-hikari.com

http://dx.doi.org/10.12988/imf.2013.36121

\title{
The Alternating Direction Difference Method Based on Variable Substitution for Telegraph Equation
}

\author{
Zongxiu Ren \\ College of Mathematics and Information Science \\ Henan Normal University, Henan Province, 453007, China \\ renzx@htu.cn \\ Guixiang Yang \\ College of Mathematics and Information Science \\ Henan Normal University, Henan Province, 453007, China \\ yangguixiang1989@163.com
}

Copyright (c) 2013 Zongxiu Ren and Guixiang Yang. This is an open access article distributed under the Creative Commons Attribution License, which permits unrestricted use, distribution, and reproduction in any medium, provided the original work is properly cited.

Abstract. In this paper, a alternating direction difference method based on variable substitution is presented for telegraph equation in the bounded domain, the theocratical analysis show that the scheme is convergence, and we get the discrete $L^{2}$-norm errors in both the approximate solution and its first derivatives for all uniform grids. 
Keywords: telegraph equation; alternating direction difference method; error estimates

\section{INTRODUCTION}

Telegraph equations as a classic mathematical physics equation it is not only of great significance but also has wide application in communication engineering. The research of telegraph equations has gained drastic attention and obtained fruitful achievements for its strong practical background. However, what most of the research adopted was the finite element method and little of that was reported on the alternating direction difference of telegraph equations. In this paper, a new approach of alternating direction difference method was employed to construct the difference scheme of telegraph equations. Due to its high convergence and stability the calculation can be simple, and convenient and the error estimation between the approximation solution and exact solution can be obtained as well.

Consider the following initial-boundary value problem of telegraph equation

$$
\begin{array}{lc}
u_{t t}-\Delta u+\alpha u_{t}+\lambda u=f(x, y, t), & (x, y, t) \in \Omega \times(0, T] \\
u(x, y, 0)=u_{0}(x, y), u_{t}(x, y, 0)=v_{0}(x, y), & (x, y) \in \Omega \\
u(x, y, t)=0, & (x, y) \in \partial \Omega, t \in(0, T]
\end{array}
$$

where $\alpha=\alpha(x, y, t), \lambda=\lambda(x, y, t), \Omega$ is a bounded domain in $R^{2}, \partial \Omega$ is the corresponding border, $T$ is a positive constant, $u_{0}(x, y), v_{0}(x, y)$ are functions have been given, for $\alpha(x, y, t), \lambda(x, y, t)$ the following conditions are established

(i) there exist $\alpha_{0}, \alpha_{1}, \lambda_{0}, \lambda_{1}$, s.t. $0<\alpha_{0} \leq \alpha(x, y, t) \leq \alpha_{1}, 0<\lambda_{0} \leq$ $\lambda(x, y, t) \leq \lambda_{1}$.

(ii) $f(x, y, t), u(x, y, t)$ are properly smooth.

Let $p(x, y, t)=\frac{\partial u(x, y, t)}{\partial t}$,

So that (1) is equivalent to

$$
p_{t}-\Delta u+\alpha p+\lambda u=f(x, y, t), \quad(x, y, t) \in \Omega \times(0, T] .
$$

\section{PRELIMINARIES}

We suppose $\Omega=(0,1) \times(0,1)$, decompose it into uniform grid , put $\Delta x=$ $\Delta y=h=\frac{1}{J}$ as space step, $\Delta t=\tau=\frac{T}{N}$ as time step, with $J, N$ are positive 
integer, note $x_{i}=i h, y_{j}=j h, i, j=0,1, \cdots, J, t^{n}=n \cdot \tau, n=0,1, \ldots, N, g_{i, j}^{n}=$ $g\left(x_{i}, y_{j}, t^{n}\right)$. Further, for convenience, let us denote

$$
\begin{gathered}
\delta_{x} U_{i, j}^{n}=\frac{U_{i+1, j}^{n}-U_{i, j}^{n}}{h}, \delta_{t} U_{i, j}^{n}=\frac{U_{i, j}^{n+1}-U_{i, j}^{n}}{\tau}, \\
\delta_{x}^{2} U_{i, j}^{n}=\frac{U_{i+1, j}^{n}-2 U_{i, j}^{n}+U_{i-1, j}^{n}}{h^{2}}, \delta_{t}^{2} U_{i, j}^{n}=\frac{U_{i, j}^{n+1}-2 U_{i, j}^{n}+U_{i, j}^{n-1}}{\tau^{2}} \\
\Delta_{h}=\delta_{x}^{2}+\delta_{y}^{2}, \nabla_{h}=\left\{\delta_{x}, \delta_{y}\right\} .
\end{gathered}
$$

Define the following Grid function

$$
S=\left\{f_{i, j} \mid f_{i, j}=f\left(x_{i}, y_{j}\right), i, j=0, \ldots, J\right\} .
$$

the discrete inner product and norm

$$
\begin{gathered}
(u, v)=\sum_{i, j=1}^{J-1} u_{i, j} v_{i, j} h^{2},\|u\|^{2}=(u, u) . \\
\left(\nabla_{h} u, \nabla_{h} v\right)=\sum_{i, j=0}^{J-1} \delta_{x} u_{i, j} \delta_{x} v_{i, j} h^{2}+\sum_{i, j=0}^{J-1} \delta_{y} u_{i, j} \delta_{y} v_{i, j} h^{2} . \\
\left\|\nabla_{h} u\right\|^{2}=\left(\nabla_{h} u, \nabla_{h} u\right)=|u|_{1 x}^{2}+|u|_{1 y}^{2} .
\end{gathered}
$$

\section{ALTERNATING DIRECTION DIFFERENCE SCHEME AND ERROR ESTIMATES}

The alternating direction difference scheme of problem $(1)-(3)$ is find $\left(U^{n}, P^{n}\right) \in S \times S$ s.t.

$$
\begin{aligned}
& \delta_{t} P_{i, j}^{n}-\triangle_{h} U_{i, j}^{n+1}+\alpha_{i, j}^{n} P_{i, j}^{n}+\lambda_{i, j}^{n} U_{i, j}^{n}+\tau^{3} \delta_{x}^{2} \delta_{y}^{2}\left(P_{i, j}^{n+1}-P_{i, j}^{n}\right)=f_{i, j}^{n}, \\
& \delta_{t} U_{i, j}^{n}=P_{i, j}^{n+1}, \\
& U_{i, j}^{0}=u_{0}\left(x_{i}, y_{j}\right), \quad P_{i, j}^{0}=\left(u_{t}\right)_{i, j}^{0}=v_{0}\left(x_{i}, y_{j}\right), \\
& U_{0, j}^{n}=U_{1, j}^{n}=U_{j, 0}^{n}=U_{j, 1}^{n}=0 .
\end{aligned}
$$

where $\tau^{3} \delta_{x}^{2} \delta_{y}^{2}\left(P_{i, j}^{n+1}-P_{i, j}^{n}\right)$ is an additional dissipation term.

Substituting (6) into (5), we have

$$
\left(I-\tau^{2} \delta_{x}^{2}\right)\left(I-\tau^{2} \delta_{y}^{2}\right) P_{i, j}^{n+1}=\left(I-\tau \alpha_{i, j}^{n}\right) P_{i, j}^{n}-\tau \lambda_{i, j}^{n} U_{i, j}^{n}+\tau\left(\delta_{x}^{2}+\delta_{y}^{2}\right) U_{i, j}^{n}+\tau^{4} \delta_{x}^{2} \delta_{y}^{2} P_{i, j}^{n}+\tau f_{i, j}^{n}
$$

introduce a new variable $P_{i, j}^{n *}$ and hence

$$
\begin{aligned}
& \left(I-\tau^{2} \delta_{x}^{2}\right) P_{i, j}^{n *}=\left(I-\tau \alpha_{i, j}^{n}\right) P_{i, j}^{n}-\tau \lambda_{i, j}^{n} U_{i, j}^{n}+\tau\left(\delta_{x}^{2}+\delta_{y}^{2}\right) U_{i, j}^{n}+\tau^{4} \delta_{x}^{2} \delta_{y}^{2} P_{i, j}^{n}+\tau f_{i, j}^{n}, \\
& \left(I-\tau^{2} \delta_{y}^{2}\right) P_{i, j}^{n+1}=P_{i, j}^{n *} \\
& \delta_{t} U_{i, j}^{n}=P_{i, j}^{n+1}
\end{aligned}
$$


where $I$ is unit operator.

The alternating direction schemes can turn multi-dimensional problem into one-dimensional problem and iterative matrix of $x, y$ are triple diagonal matrices which have same structure, so they are easy to program for computing.

At the point $\left(x_{i}, y_{j}, t^{n}\right),(4)$ and $p$ can be written as

$$
\begin{aligned}
& \delta_{t} p_{i, j}^{n}-\triangle_{h} u_{i, j}^{n+1}+\alpha_{i, j}^{n} p_{i, j}^{n}+\lambda_{i, j}^{n} u_{i, j}^{n}+\tau^{3} \delta_{x}^{2} \delta_{y}^{2}\left(p_{i, j}^{n+1}-p_{i, j}^{n}\right)=f_{i, j}^{n}+R_{1}, \\
& \delta_{t} u_{i, j}^{n}=p_{i, j}^{n+1}+R_{2} .
\end{aligned}
$$

where

$$
\begin{aligned}
R_{1} & =\delta_{t} p_{i, j}^{n}-\left(p_{t}\right)_{i, j}^{n}+(\Delta u)_{i, j}^{n}-\triangle_{h} u_{i, j}^{n}+\triangle_{h} u_{i, j}^{n}-\triangle_{h} u_{i, j}^{n+1}+\tau^{3} \delta_{x}^{2} \delta_{y}^{2}\left(p_{i, j}^{n+1}-p_{i, j}^{n}\right) \\
& =R_{11}+R_{12}+R_{13}+R_{14} . \\
R_{2} & =\delta_{t} u_{i, j}^{n}-\left(u_{t}\right)_{i, j}^{n}+p_{i, j}^{n}-p_{i, j}^{n+1} \\
& =R_{21}+R_{22} .
\end{aligned}
$$

Set $e=u-U, \theta=p-P$, Subtracting (5)(6) from (9)(10), we obtain the error equations

$$
\begin{aligned}
& \delta_{t} \theta_{i, j}^{n}-\triangle_{h} e_{i, j}^{n+1}+\alpha \theta_{i, j}^{n}+\lambda e_{i, j}^{n}+\tau^{3} \delta_{x}^{2} \delta_{y}^{2}\left(\theta_{i, j}^{n+1}-\theta_{i, j}^{n}\right)=R_{1}, \\
& \delta_{t} e_{i, j}^{n}=\theta_{i, j}^{n+1}+R_{2} .
\end{aligned}
$$

Make inner product with $\theta^{n+1}$ on both sides of (14), and hence

$$
\begin{aligned}
& \left(\delta_{t} \theta^{n}, \theta^{n+1}\right)-\left(\triangle_{h} e^{n+1}, \delta_{t} e^{n}\right)+\tau^{3}\left(\delta_{x}^{2} \delta_{y}^{2}\left(\theta^{n+1}-\theta^{n}\right), \theta^{n+1}\right)+\left(\lambda^{n} e^{n}, \theta^{n+1}\right) \\
& =\left(R_{1}, \theta^{n+1}\right)-\left(\alpha^{n} \theta^{n}, \theta^{n+1}\right)-\left(\triangle_{h} e^{n+1}, R_{2}\right) .
\end{aligned}
$$

Now we will estimate one by one.

$$
\begin{aligned}
\left(\delta_{t} \theta^{n}, \theta^{n+1}\right) \geq & \frac{1}{2 \tau}\left(\left\|\theta^{n+1}\right\|^{2}-\left\|\theta^{n}\right\|^{2}\right) \\
-\left(\triangle_{h} e^{n+1}, \delta_{t} e^{n}\right) & =\frac{1}{\tau} \sum_{i=0}^{J-1} \sum_{j=1}^{J-1} \delta_{x} e_{i, j}^{n+1} \delta_{x}\left(e_{i, j}^{n+1}-e_{i, j}^{n}\right) h^{2}+\frac{1}{\tau} \sum_{i=0}^{J-1} \sum_{j=1}^{J-1} \delta_{y} e_{i, j}^{n+1} \delta_{y}\left(e_{i, j}^{n+1}-e_{i, j}^{n}\right) h^{2} \\
& \geq \frac{1}{2 \tau} \sum_{i=0}^{J-1} \sum_{j=0}^{J-1}\left(\delta_{x} e_{i, j}^{n+1}\right)^{2} h^{2}-\frac{1}{2 \tau} \sum_{i=0}^{J-1} \sum_{j=0}^{J-1}\left(\delta_{x} e_{i, j}^{n}\right)^{2} h^{2} \\
+ & \frac{1}{2 \tau} \sum_{i=0}^{J-1} \sum_{j=0}^{J-1}\left(\delta_{y} e_{i, j}^{n+1}\right)^{2} h^{2}-\frac{1}{2 \tau} \sum_{i=0}^{J-1} \sum_{j=0}^{J-1}\left(\delta_{y} e_{i, j}^{n}\right)^{2} h^{2} \\
& =\frac{1}{2 \tau}\left(\left\|\nabla_{h} e^{n+1}\right\|^{2}-\left\|\nabla_{h} e^{n}\right\|^{2}\right) . \\
\tau^{3}\left(\delta_{x}^{2} \delta_{y}^{2}\left(\theta^{n+1}-\theta^{n}\right), \theta^{n+1}\right) & =\tau^{3}\left(\delta_{x} \delta_{y} \theta^{n+1}, \delta_{x} \delta_{y} \theta^{n+1}\right)-\tau^{3}\left(\delta_{x} \delta_{y} \theta^{n}, \delta_{x} \delta_{y} \theta^{n+1}\right) \\
& \geq \tau^{3}\left\|\delta_{x} \delta_{y} \theta^{n+1}\right\|^{2}-\frac{\tau^{3}}{2}\left(\left\|\delta_{x} \delta_{y} \theta^{n+1}\right\|^{2}+\left\|\delta_{x} \delta_{y} \theta^{n}\right\|^{2}\right) \\
& =\frac{\tau^{3}}{2}\left(\left\|\delta_{x} \delta_{y} \theta^{n+1}\right\|^{2}-\left\|\delta_{x} \delta_{y} \theta^{n}\right\|^{2}\right) .
\end{aligned}
$$


By $\varepsilon$ - inequality and $\lambda$ is bounded, we have

$$
\left(\lambda^{n} e^{n}, \theta^{n+1}\right) \leq \frac{\lambda_{1}^{2}}{4 \varepsilon}\left\|e^{n}\right\|^{2}+\varepsilon\left\|\theta^{n+1}\right\|^{2} .
$$

Then by Holder inequality and $\varepsilon$ - inequality, we obtain

$$
\begin{aligned}
\left(R_{11}, \theta^{n+1}\right) & =\sum_{i=1}^{J-1} \sum_{j=1}^{J-1} \frac{1}{\tau} \int_{t^{n}}^{t^{n+1}}\left(t^{n+1}-s\right) \frac{\partial^{2} p_{i, j}}{\partial s^{2}} d s \cdot \theta_{i, j}^{n+1} \cdot h^{2} \\
& \leq \sum_{i=0}^{J-1} \sum_{j=0}^{J-1} \frac{1}{\tau}\left(\left[\int_{t^{n}}^{t^{n+1}}\left(\frac{\partial^{2} p_{i, j}}{\partial s^{2}}\right)^{2} d s\right]^{\frac{1}{2}} \cdot\left[\int_{t^{n}}^{t^{n+1}}\left(t^{n+1}-s\right)^{2} d s\right]^{\frac{1}{2}}\right) \cdot \theta_{i, j}^{n+1} \cdot h^{2} \\
& \leq C\left\|\theta^{n+1}\right\|^{2}+C \tau\left\|p_{t t}\right\|_{L^{2}\left(t^{n}, t^{n+1} ;\|\cdot\|\right)}^{2} \\
\left(R_{12}, \theta^{n+1}\right) & =\sum_{i=0}^{J-1} \sum_{j=0}^{J-1}\left[\left(\frac{\partial^{2} u}{\partial x^{2}}\right)_{i, j}^{n}+\left(\frac{\partial^{2} u}{\partial y^{2}}\right)_{i, j}^{n}-\left(\delta_{x}^{2}+\delta_{y}^{2}\right) u_{i, j}^{n}\right] \cdot \theta_{i, j}^{n+1} \cdot h^{2} \\
& \leq \sum_{i=0}^{J-1} \sum_{j=0}^{J-1} C \cdot \frac{1}{h^{2}}\left[\int_{x_{i}}^{x_{i+1}}\left(x_{i+1}-s\right)^{2} \frac{\partial^{3} u_{i, j}}{\partial s^{3}} d s+\int_{y_{j}}^{y_{j+1}}\left(y_{i+1}-s\right)^{2} \frac{\partial^{3} u_{i, j}}{\partial s^{3}} d s\right] \cdot \theta_{i, j}^{n+1} \cdot h^{2} \\
& \leq C h^{4}\left(\left\|\frac{\partial^{3} u}{\partial x^{3}}\right\|^{2}+\left\|\frac{\partial^{3} u}{\partial y^{3}}\right\|^{2}\right)+C\left\|\theta^{n+1}\right\|^{2} .
\end{aligned}
$$

and similarly

$$
\begin{aligned}
\left(R_{13}, \theta^{n+1}\right) \leq C h^{4}( & \left.\left\|\frac{\partial^{3} u}{\partial x^{2} \partial t}\right\|_{L^{2}\left(t^{n}, t^{n+1} ;\|\cdot\|\right)}^{2}+\left\|\frac{\partial^{3} u}{\partial y^{2} \partial t}\right\|_{L^{2}\left(t^{n}, t^{n+1} ;\|\cdot\|\right)}^{2}\right)+C\left\|\theta^{n+1}\right\|^{2} . \\
\left(R_{14}, \theta^{n+1}\right) & =\tau^{3} \sum_{i=1}^{J-1} \sum_{j=1}^{J-1} \delta_{x}^{2} \delta_{y}^{2}\left(p_{i, j}^{n+1}-p_{i, j}^{n}\right) \cdot \theta_{i, j}^{n+1} \cdot h^{2} \\
& =\tau^{3} \sum_{i=0}^{J-1} \sum_{j=0}^{J-1} \int_{t^{n}}^{t^{n+1}} \delta_{x} \delta_{y}\left(\frac{\partial p_{i, j}}{\partial s}\right) d s \cdot \delta_{x} \delta_{y} \theta_{i, j}^{n+1} \cdot h^{2} \\
& \leq C \tau^{3}\left\|\delta_{x} \delta_{y} \theta^{n+1}\right\|^{2}+C \tau^{3}\left\|\frac{\partial^{3} p}{\partial x \partial y \partial t}\right\|_{L^{2}\left(t^{n}, t^{n+1} ;\|\cdot\|\right)}^{2} .
\end{aligned}
$$

By $\varepsilon$ - inequality and $\alpha$ is bounded, we have

$$
\left|-\left(\alpha^{n} \theta^{n}, \theta^{n+1}\right)\right| \leq \varepsilon\left\|\theta^{n+1}\right\|^{2}+\frac{\alpha_{1}^{2}}{4 \varepsilon}\left\|\theta^{n}\right\|^{2} .
$$

By Taylor expansion and Holder inequality, we get

$$
\begin{aligned}
-\left(\triangle_{h} e^{n+1}, R_{2}\right) & =\sum_{i=1}^{J-1} \sum_{j=1}^{J-1} \nabla_{h}\left(\frac{1}{\tau} \int_{t^{n}}^{t^{n+1}}\left(t^{n+1}-s\right) \frac{\partial^{2} u_{i, j}}{\partial s^{2}} d s\right) \cdot \nabla_{h} e_{i, j}^{n+1} \cdot h^{2} \\
& +\sum_{i=1}^{J-1} \sum_{j=1}^{J-1} \nabla_{h}\left(\int_{t^{n}}^{t^{n+1}} \frac{\partial p_{i, j}}{\partial s} d s\right) \cdot \nabla_{h} e_{i, j}^{n+1} \cdot h^{2} \\
& \leq C \tau h^{2}\left(\left\|\frac{\partial^{3} u}{\partial x \partial t^{2}}\right\|_{L^{2}\left(t^{n}, t^{n+1} ;\|\cdot\|\right)}^{2}+\left\|\frac{\partial^{3} u}{\partial y \partial t^{2}}\right\|_{L^{2}\left(t^{n}, t^{n+1} ;\|\cdot\|\right.}^{2}+\left\|\frac{\partial^{2} p}{\partial y \partial t}\right\|_{L^{2}\left(t^{n}, t^{n+1} ;\|\cdot\|\right.}^{2}+C\left\|\nabla_{h} e^{n+1}\right\|^{2} .\right. \\
& +\left\|\frac{\partial^{2} p}{\partial x \partial t}\right\|_{L^{2}\left(t^{n}, t^{n+1} ;\|\cdot\|\right)}^{2}
\end{aligned}
$$


Substituting (17) - (22) into (16), and sum on $n$ from 1 to $N-1$, we obtain

$$
\begin{aligned}
& \left\|\theta^{N}\right\|^{2}-\left\|\theta^{0}\right\|^{2}+\left\|\nabla_{h} e^{N}\right\|^{2}-\left\|\nabla_{h} e^{0}\right\|^{2}+\tau^{4}\left(\left\|\delta_{x} \delta_{y} \theta^{N}\right\|^{2}-\left\|\delta_{x} \delta_{y} \theta^{0}\right\|^{2}\right) \\
& \leq C \tau \sum_{n=0}^{N-1}\left\|\theta^{n+1}\right\|^{2}+C \tau^{2}\left(\left\|p_{t t}\right\|_{L^{2}(0, T ;\|\cdot\|)}^{2}+h^{4}\left\|\frac{\partial^{3} u}{\partial x \partial y \partial t}\right\|_{L^{2}(0, T ;\|\cdot\|)}^{2}\right)+C \tau^{4}\left(\sum_{n=0}^{N-1}\left\|\delta_{x} \delta_{y} \theta^{n+1}\right\|^{2}\right. \\
& \left.+\left\|\frac{\partial^{3} p}{\partial x \partial y \partial t}\right\|_{L^{2}(0, T ;\|\cdot\|)}^{2}\right)+C \tau^{2} h^{2}\left(\left\|\frac{\partial^{3} u}{\partial x \partial t^{2}}\right\|_{L^{2}(0, T ;\|\cdot\|)}^{2}+\left\|\frac{\partial^{3} u}{\partial y \partial t^{2}}\right\|_{L^{2}(0, T ;\|\cdot\|)}^{2}\right. \\
& \left.+\left\|\frac{\partial^{2} p}{\partial x \partial t}\right\|_{L^{2}(0, T ;\|\cdot\|)}^{2}+\left\|\frac{\partial^{2} p}{\partial y \partial t}\right\|_{L^{2}(0, T ;\|\cdot\|)}^{2}\right)+C \tau \sum_{n=0}^{N-1}\left\|\nabla_{h} e^{n+1}\right\|^{2} .
\end{aligned}
$$

Choose properly $\tau$ s.t. $C \tau \leq \frac{1}{2}$, then

$$
\begin{aligned}
& \left\|\theta^{N}\right\|^{2}+\left\|\nabla_{h} e^{N}\right\|^{2}+\tau^{4}\left\|\delta_{x} \delta_{y} \theta^{N}\right\|^{2} \leq\left\|\theta^{0}\right\|^{2}+\left\|\nabla_{h} e^{0}\right\|^{2}+\tau^{4}\left\|\delta_{x} \delta_{y} \theta^{0}\right\|^{2} \\
& +C \tau \sum_{n=0}^{N-1}\left\|\theta^{n}\right\|^{2}+C \tau \sum_{n=0}^{N-1}\left\|\nabla_{h} e^{n}\right\|^{2}+C \tau^{4} \sum_{n=0}^{N-1}\left\|\delta_{x} \delta_{y} \theta^{n+1}\right\|^{2} \cdot \tau+C\left(\tau^{2}+\tau^{2} h^{2}+h^{4}\right) .
\end{aligned}
$$

By discrete Gronwall inequality, we obtain

$$
\left\|\theta^{N}\right\|^{2}+\left\|\nabla_{h} e^{N}\right\|^{2}+\tau^{4}\left\|\delta_{x} \delta_{y} \theta^{N}\right\|^{2} \leq C\left(\tau^{2}+\tau^{2} h^{2}+h^{4}\right) .
$$

Theorem 3.1. Let $u$ and $p$ are the solution of (1) - (3) and (4) respectively, $U^{n}$ and $P^{n}$ are the solution of $(5)-(6)$ respectively, assume that the grid in the solving area is equidistant subdivision, then there exists a constant $C$ independent $h, \tau$, s.t. for any $0 \leq n \leq N\left(N \leq\left[\frac{T}{\tau}\right]\right)$ we have

$$
\left\|p^{n}-P^{n}\right\|^{2}+\left\|\nabla_{h}\left(u^{n}-U^{n}\right)\right\|^{2} \leq C\left(\tau^{2}+\tau^{2} h^{2}+h^{4}\right) .
$$

\section{REFERENCES}

[1] Mawhin J, Orteg R, Robles-Perez A M.Maximum principles for bounded solutions of the telegraph equation in space dimensions two and three and applications. Differential Equations, 2005, 208(1), 42-63.

[2] G.Ma and J.Q.Zhou. Telegraph equation $H$ - Galerkin the coordination of mixed finite element analysis. Mathematics in Practice and Theory, 2010,40(16), 107-112.

[3] Y.Z.Zhang. High-dimensional telegraph equation symmetric periodic solution. Acta Analysis Functionalis Applicate, 2004,6(3), 236-239.

[4] J.W.Hu and H.M.Tang. Numerical methods of differential equations. Beijing, Since Press, 2003.

\section{Received: June 13, 2013}

